\title{
A Luminex based serological assay for detecting IgM and IgG antibody response in SARS-CoV2 patients
}

\author{
Shailendra Mani ${ }^{1}$, Tripti Shrivastava ${ }^{1}$, Sankar Bhattacharyya ${ }^{1}$, Nagender Rameshwaram \\ ${ }^{1}$, Sweety Samal ${ }^{1}$, Sandeep Goswami ${ }^{1}$, Ashok Kumar ${ }^{1}$, Ramandeep Singh ${ }^{1}$, Niraj Kumar ${ }^{1}$, \\ Nitya Wadhwa ${ }^{1}$, Uma Chandra Natchu ${ }^{1}$, Ramachandran Thiruvengadam ${ }^{1}$, Shailaja \\ Sopory ${ }^{1}$, Pallavi Kshetrapal ${ }^{1}$, Bapu Desiraju ${ }^{1}$, and Vandita Bhartia ${ }^{1}$ \\ ${ }^{1}$ Translational Health Science and Technology Institute
}

June 23, 2020

\begin{abstract}
Nucleic acid real-time PCR test has become the main diagnostic tool for SARS-CoV-2 infection, and there are also drawbacks to these real-time PCR test kits. Additionally, it has reported high false-negative rates. A reliable and rapid test method is urgently required to briskly detect antibody response in a large number of infected patients and ensure prompt care for patients. Therefore, we report here a Luminex bead based multiplexed immunoassay to serologically diagnose the SARS-CoV2 infection and detect IgM and IgG response in COVID-19 patients. The technology can provide sensitive and specific detection using a low amount of serum samples in high-throughput screening platforms.
\end{abstract}

\section{Introduction}

The recent global outbreak of COVID-19 caused by SARS-CoV2, a member of coronavirus family originated from China (Wuhan, Hubei province) imposed catastrophic impacts on every society, infecting at least 8.79 million people worldwide resulting in 0.46 million deaths as of today [1- 4]. There are several reasons for this pervasive problem; however, there is scarce information on the assessment and diagnosis of patients infected with COVID-19, and the potential risk of respiratory illness which may be mild or severe. The current approach to diagnose the viral infection is based on molecular detection using RT-PCR assay which detects the viral load in clinical samples. However, as a consequence of low virus titer, there have been instances when the test has failed to detect the viral RNA from clinical samples [5].

SARS-CoV-2 antibodies in patient blood is a reasonable choice for immediate, quick, highly responsive diagnosis of COVID-19. It is commonly agreed that immunoglobulin M (IgM) offers the first line of protection during viral infections prior to the generation of adaptive, high-affinity IgG responses that are essential for long-term immune and immunological memory [6]. We believe that the mechanism of antibody production against SARS-CoV-2 would be an indicator of infection. Hence, to ameliorate the current diagnosis, in this article we report a bead-based multiplex immunoassay to detect IgG and IgM responses from the serum samples of COVID-19 patients. This would eventually allow for a deeper analysis, as antibodies persist in serum for a longer period of time and henceforth increase the chances for detection, which would benefit from adopting Luminex based serology assay as a routine diagnostic technique for SARS-CoV2 infection.

We sampled 20 clinical specimens that were symptomatic and RT-PCR tested, from the total of cases reported to the THSTI-Biorepository collected under the DBT India consortium for the COVID-19 research program and IRB approvals from all partnering clinical sites to conduct this study. The 20 clinical specimens which were either positive by RT-PCR or suspected from the hospital (data not shown) were tested using the Luminex platform against the Receptor-Binding Domain (RBD) and Spike antigens of SARS-CoV2 
and 10 clinical specimens collected pre-pandemic from enrolled participants were used as negative controls. To develop the aforementioned Luminex based serology assay capable of detecting COVID-19 infection, we expressed RBD and Spike proteins and purified using a mammalian expression system and metal affinity chromatography. Both the purified proteins were coupled separately on Luminex beads as described previously [7] and confirmed by Western blot using the anti-His-HRP antibody. Aiming to unveil specificity of the assay, strict cut-off (5X SD + Mean) was used for RBD and spike proteins to rule out any non-specific readings.

Our results showed a higher antibody reaction to spike protein relative to RBD (Table I), therefore decided to continue using only spike protein for future tests. The developed Luminex platform against the RBD and spike protein was able to detect both IgG and IgM responses in patient serum samples (Figure 1 \& Table I). Precisely, we were able to detect IgM and IgG response for 11 out of 12 patient serum samples (Table I) that tested positive by RT-PCR, with an exception of patient \# 16 for the IgM response to the Luminex assay. Contrarily the patient \# 18 which was earlier tested RT-PCR positive and showed high IgM response by Luminex did not show the IgG response. Moreover, patient \# 1 and \# 6 which were IgM negative showed IgG antibody responses. Apart from these few exceptions, IgM and IgG were detected in most of the analyzed samples by Luminex assay (Figure 1 \& Table I), suggesting that the assay could be a strategy for the adoption of manageable and scalable research practices for community screening during COVID-19.

In addition to the diagnostic benefit, our results showed a clear positive association between clinical seriousness and $\mathrm{Ab}$ titer in the COVID-19 patients. Whereas the causal association between hormonal reaction and seriousness of the disease was still uncertain, the findings pose a theoretical use of the strong $\mathrm{Ab}$ titer as a proxy predictor for bad clinical prognosis. As most of the recovering patients encountered with viral infections show the presence of neutralizing antibodies (NAbs), which are strong predictors of defensive immunity. We further patrolled the results of the serology platform to determine the presence of NAbs in the patient serum samples. All the samples were heat-inactivated at $56^{\circ} \mathrm{C}$ for $1 \mathrm{~h}$ before testing with the SARS-CoV2 virus [8]. The virus was obtained from the BEI resources (Isolate USA-WA1/2020) [9], passaged once in Vero cells, titrated and $1 \times 10^{2}$ TCID $_{50}$ virus (diluted in $50 \mu$ of the serum-free media) was incubated with 2.0 fold serial dilutions for 90 mins followed by $1 \mathrm{~h}$ adsorption on Vero cells. The cells were subsequently washed and DMEM media supplemented with $2 \%$ FBS was added to cells. The presence of cytopathic effect $(\mathrm{CPE})$ was observed in cells using a microscope after incubation for $4-5$ days at $37^{\circ} \mathrm{C}$ with $5 \% \mathrm{CO}_{2}$. We found that, following infection, antibodies were elicited against the spike protein and when tested in a serum neutralization assay these sera were able to neutralize SARS-CoV-2. Results from serum neutralization experiments revealed, 7/11 IgM and 7/13 IgG with the presence of NAbs against SARS-CoV2 virus in the serology positive samples. The neutralizing antibody titers were observed in the range between 1:20 (\# 11 \& \# 17) and 1:320 (\# 9). However, as expected, we did not observe any Luminex IgM/IgG response or neutralization antibodies in healthy/negative participant sera (Table I). This part of the study validates the Luminex assay with a similar set of samples and provides scope for expansion on a larger platform.

In summary, the identification of both IgM and IgG may provide details on the timing of the virus infection. The fast identification of both IgM and IgG antibodies would bring importance to COVID-19 disease diagnosis and care. Hence our approach for the COVID-19 serology problem with an alternative method that enables detecting viral antigen-specific antibodies with enhanced specificity and sensitivity in a simple, safe, fast and inexpensive manner might resolve this long-standing question for the faster diagnosis of SARS-CoV2. A correlative study with the early production of neutralizing antibodies in the samples of patients tested by Luminex assay was also documented. The developed assay after a systematic validation with an adequate sample size could be utilized for large scale community screening, screening for the potential plasma donors, and evaluating the efficacy of the future vaccine candidate trials.

\section{References}

1. World Health Organization. Novel coronavirus-China [cited 2020 Jan 12 . https://www.who.int/csr/don/12-january-2020-novel-coronavirus-china/en/ 
2. World Health Organization. Media briefing on COVID-19 -11 March 2020 https://www.who.int/dg/speeches/detail/who-director-general-s-opening-remarks-at-the-mediabriefing-on-covid-19-11-march-2020

3. WHO Coronavirus Disease (COVID-19) Dashboard; https://covid19.who.int

4. Centers for Disease Control and Prevention. Symptoms of Coronavirus https://www.cdc.gov/coronavirus/2019-ncov/symptoms-testing/symptoms.html

5. Xie X, Zhong Z, Zhao W, et al. Chest CT for typical 2019-nCoV pneumonia: Relationship to negative RT-PCR testing. doi:10.1148/radiol.202020 0343200343.

6. Racine R, Winslow GM. IgM in microbial infections: taken for granted? Immunol Lett. 2009;125(2):7985.

7. Mani S, Tan CW, Wang LF, et al. Serological cross reactivity between zika and dengue viruses in experimentally infected monkeys. doi: 10.1007/s12250-018-0048-8.

8. Zhou P, Yang XL, Wang XG, et al. A pneumonia outbreak associated with a new coronavirus of probable bat origin. Nature. 2020 Feb. doi:10.1038/s41586-020-2012-7

9. Harcourt J, Tamin A, Lu X, et al. Severe acute respiratory syndrome coronavirus 2 from patient with 2019 novel coronavirus disease, United States.

Table 1: Serum neutralization titers of patients samples

\begin{tabular}{|c|c|c|c|c|c|c|}
\hline Patient No. & Samples Id & RT-PCR & Duration of days of symptoms & Luminex assay & Luminex assay & Luminex a \\
\hline & & & & $\operatorname{IgM}$ & $\operatorname{IgM}$ & $\operatorname{Ig} G$ \\
\hline & & & & $\mathrm{RBD}$ & Spike & $\mathrm{RBD}$ \\
\hline Cut off & & & & 484.93 & 465.01 & 32.66 \\
\hline \# 1 & C3020001120201 & Negative & NA & 469.75 & 310 & 62.75 \\
\hline \# 2 & C3020002120201 & Negative & 22 & 423.75 & 345.75 & 14 \\
\hline$\# 3$ & C3020003120201 & Negative & 2 & 364.75 & 223.25 & 26.25 \\
\hline \# 4 & C3020004120201 & Positive & 0 & 470 & 849.5 & 27 \\
\hline$\# 5$ & C3020005110201 & Negative & 4 & 164.75 & 206.25 & 20.75 \\
\hline \# 6 & C3020006110201 & Negative & 16 & 284 & 242.5 & 31 \\
\hline \# 7 & C3020007110201 & Negative & 9 & 28.75 & 24 & 19.5 \\
\hline \# 8 & C3030001110201 & Positive & 10 & 405.5 & 5667 & 24.5 \\
\hline \# 9 & C3030002110201 & Positive & NA & 717 & 6133.25 & 113.5 \\
\hline \# 10 & C3030003110201 & Positive & 12 & 995.75 & 5692.5 & 91.25 \\
\hline \# 11 & C3030004110201 & Positive & 14 & 503.75 & 1505 & 27 \\
\hline \# 12 & C3030005110201 & Positive & 18 & 269.25 & 1132.25 & 20.5 \\
\hline \# 13 & C3030006110201 & Positive & 12 & 363.25 & 2553.5 & 33 \\
\hline \# 14 & C3030007110201 & Positive & 11 & 208.75 & 1147.25 & 24 \\
\hline \# 15 & C3030008110201 & Positive & 11 & 167 & 1045.5 & 19.5 \\
\hline \# 16 & C3030009110201 & Positive & 12 & 143.75 & 394.75 & 21.5 \\
\hline \# 17 & C3030010110201 & Positive & 13 & 238 & 2209.75 & 27.5 \\
\hline \# 18 & C3030011110201 & Positive & 7 & 290.75 & 1112.75 & 23.5 \\
\hline \# 19 & C3040001110201 & Negative & 7 & 380.25 & 214 & 27 \\
\hline \# 20 & C3040002110201 & Negative & 7 & 145.25 & 251.5 & 20 \\
\hline \# 21 & P3027501130901 & $\mathrm{PN}$ & NA & 197 & 135 & 17.5 \\
\hline \# 22 & P3029511130901 & PN & NA & 278 & 209 & 21 \\
\hline \# 23 & P3004391130901 & PN & NA & 141 & 107.25 & 21 \\
\hline \# 24 & P3024531130901 & PN & NA & 105 & 86.5 & 16 \\
\hline \# 25 & P3013361130901 & $\mathrm{PN}$ & NA & 249.5 & 216 & 23 \\
\hline \# 26 & P3048921130901 & $\mathrm{PN}$ & NA & 182 & 313 & 19 \\
\hline \# 27 & P3027501130901 & $\mathrm{PN}$ & NA & 286.25 & 148 & 24.5 \\
\hline \# 28 & P3030431130901 & $\mathrm{PN}$ & NA & 200 & 170 & 19.5 \\
\hline \# 29 & P3047941130901 & PN & NA & 188 & 121 & 16.5 \\
\hline
\end{tabular}




\begin{tabular}{lllllll}
\hline Patient No. & Samples Id & RT-PCR & Duration of days of symptoms & Luminex assay & Luminex assay & Luminex a \\
\hline$\# 30$ & P3036761130901 & PN & NA & 147.75 & 131.5 & 19.5 \\
\hline
\end{tabular}

NA- Not available, PN- Presumed negative (pre-pandemic serum)

Figure 1: IgM and IgG antibody response against the spike protein of the SARS-CoV2 virus.

30 serum samples were tested in duplicates and mean fluorescent intensity (MFI) was plotted for each sample. $5 \mathrm{X} \mathrm{SD}+$ Mean was used to set up the cut off (red dashed line-IgM and blue dashed line- IgG) which was based on the known healthy/COVID-19 negative serum samples. Samples with no neutralization titer was defined as negative (-); with neutralization titer at serum dilution of 1:20, 1:40-1:80 and 1:160-1:320 as (+), $(++)$ and $(+++)$, respectively.

\section{Hosted file}

Figure 1.docx available at https://authorea.com/users/336148/articles/461913-a-luminexbased-serological-assay-for-detecting-igm-and-igg-antibody-response-in-sars-cov2-

patients 